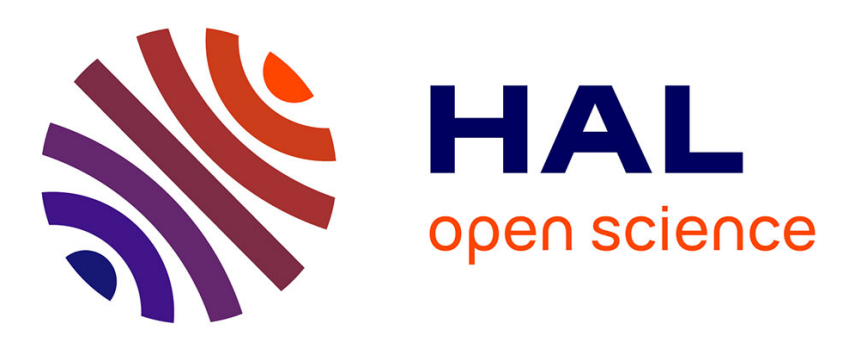

\title{
Quantitative Thermoacoustic Tomography with microwaves sources
}

Hassan Akhouayri, Maïtine Bergounioux, Anabela da Silva, Peter Elbau, Amélie Litman, Leonidas Mindrinos

\section{- To cite this version:}

Hassan Akhouayri, Maïtine Bergounioux, Anabela da Silva, Peter Elbau, Amélie Litman, et al.. Quantitative Thermoacoustic Tomography with microwaves sources. Journal of Inverse and Ill-posed Problems, 2016, 25 (6), pp.703. 10.1515/jiip-2016-0012 . hal-01267412v2

HAL Id: hal-01267412 https://hal.science/hal-01267412v2

Submitted on 4 Nov 2016

HAL is a multi-disciplinary open access archive for the deposit and dissemination of scientific research documents, whether they are published or not. The documents may come from teaching and research institutions in France or abroad, or from public or private research centers.
L'archive ouverte pluridisciplinaire HAL, est destinée au dépôt et à la diffusion de documents scientifiques de niveau recherche, publiés ou non, émanant des établissements d'enseignement et de recherche français ou étrangers, des laboratoires publics ou privés. 


\title{
Quantitative Thermoacoustic Tomography with microwaves sources*
}
H. Akhouayri ${ }^{\dagger}$
M. Bergounioux ${ }^{\ddagger}$
A. Da Silva ${ }^{\dagger}$
P. $\mathrm{Elbau}^{\S}$
A. Litman ${ }^{\dagger}$
L. Mindrinos ${ }^{\S}$

November 4, 2016

\begin{abstract}
We investigate a quantitative thermoacoustic tomography process. We aim to recover the electric susceptibility and the conductivity of a medium when the sources are in the microwaves range. We focus on the case where the source signal has a slow time-varying envelope. We present the direct problem coupling equations for the electric field, the temperature variation and the pressure (to be measured via sensors). Then, we give a variational formulation of the inverse problem which takes into account the entire electromagnetic, thermal and acoustic coupled system, and perform the formal computation of the optimality system.
\end{abstract}

Keywords Thermoacoustic tomography, inverse problem, optimal control, Maxwell's equations.

AMS Subject Classification: 35M33, 35Q61, 49N45, 80A23, 93C20

\section{Introduction}

In this paper we investigate a model to describe the so called thermoacoustic tomography process as precisely as possible, in the very case where the sources are in the microwaves range $[13,19$, $27,35]$. Let us briefly recall the principle of photo/thermoacoustic tomography (we refer to $[21,22,28,33,34]$ for a more complete description).

Photoacoustic and thermoacoustic tomographies (PAT and TAT) consist in transmitting a time-modulated electromagnetic wave (generally a pulse in the optical or near-infrared domain for PAT and in the microwave domain for TAT), whose energy is locally absorbed by the tissue absorbers, transformed into heat and then into acoustic (generally ultrasonic) waves via the thermo-expansion mechanism.

The physical model routinely used in PAT is similar to the one in TAT, apart from the fact that the reconstructions performed are based on input signals in different frequency bands. In

\footnotetext{
${ }^{*}$ This work is supported by ANR (AVENTURES - ANR-12-BLAN-BS01-0001-01) and PHC AMADEUS, OEAD WTZ FR14/2013

${ }^{\dagger}$ Aix-Marseille Université, CNRS, Centrale Marseille, Institut Fresnel UMR7249, 13013 Marseille, France

${ }^{\ddagger}$ University of d'Orléans, Laboratory MAPMO, CNRS, UMR 7349, Fédération Denis Poisson, FR 2964, Bâtiment de Mathématiques, BP 6759, 45067 Orléans cedex 2, France, maitine.bergounioux@univ-orleans.fr

${ }^{\S}$ Computational Science Center, University of Vienna, 1090 Vienna, Austria
} 
TAT, one reconstructs refraction indices and absorption coefficients, in PAT, one reconstructs diffusion and absorption coefficients.

Most of quantitative PAT methods assume that the initial pressure distribution has been reconstructed and then perform the identification of the absorption and/or diffusion coefficients. To achieve this goal one can use many measurements, for instance different lightning sources [4] or different wavelength sources [37]. Some authors assume that the Green function associated to the acoustic wave is known and perform the inversion with the pressure measurements [15, 31, 32]. Most models are based on the diffusion equation but there are some works that use the radiative transfer equation (RTE) as in [30]. The simplified model from PAT that only involves the pressure effects, does not take into account the optical inhomogeneity of the medium. It is then necessary to go one step further in the modelling of the medium, which will allow to reconstruct absorption coefficients. This has been achieved in [7] where the full coupled model has been investigated.

The aim of this paper is to focus on the TAT case and to provide a complete model coupling electromagnetic source effects, heat transfer and pressure equations, for quantitative thermoacoustic tomography. Though, the equations are well known, such a model that fully describes the whole direct problem is new to our knowledge. The heat transfer equations were first presented in the context of PAT in [10] but then neglected under the assumption of instantaneous heating. Indeed, the quantitative PAT methods, currently available, assume that the initial pressure distribution, i.e., the amount of absorbed radiation, has been first reconstructed and then perform the identification of the absorption and diffusion coefficients using mainly the diffusion approximation of the radiative transfer equation, see for example $[1,3,16,26,38]$, or the electromagnetic parameters using Maxwell's equations [12].

For quantitative reconstructions in TAT, we refer to [5] where the authors neglect the thermal effects and handle the scalar Helmholtz equation. Furthermore, they use a linearized version to deal with the inverse problem to recover the absorption coefficient. In [6], they present a similar approach for the vectorial Maxwell's equation and reconstruct the absorption coefficient and the refractive index of the materials. In [2], the Helmholtz equation is considered and an optimal control algorithm is proposed to recover the absorption coefficient from the local knowledge of the absorbed radiation. An iterative scheme, using the Helmholtz equation, to reconstruct the conductivity is also presented in [17].

In this paper, we present the direct problem, where the thermal effects are fully kept. Nevertheless, we focus on the very case where the source pulse has a slowly time varying envelope, which is the most relevant situation from a practical point of view $[24,36]$. We also propose a way to solve the corresponding quantitative inverse problem using a variational formulation as in [7], thus taking into account the complete coupled model. The paper is organized as follows: we first set the description of the different processes, namely the electromagnetic, heating and acoustic ones. Then, we specify the mathematical model and present the equivalent minimization problem. The paper ends with the (formal) optimality conditions for the discretized system of equations. 


\section{The Thermoacoustic model}

\subsection{Electromagnetic propagation}

To describe the propagation of the electromagnetic waves due to an illumination by a radiofrequency source, we consider the classical macroscopic Maxwell's equations (in SI units) and the constitutive relations between the electric displacement $D$, the electric field $E$, the magnetic induction $B$ and the magnetic field $H$. All these vectors are three-dimensional real-valued functions of position $x$ and time $t$.

\subsubsection{Maxwell's equations}

We assume that the medium is non-magnetic, linear dielectric, isotropic and inhomogeneous. Let $\Omega \subset \mathbb{R}^{3}$ be a bounded domain with smooth boundary such that the exterior domain $\mathbb{R}^{3} \backslash \Omega$ is connected. The domain $\Omega$ contains the support of the inhomogeneity we aim at recovering with the TAT technique. This domain may be possibly disconnected.

In the sequel,

- the external current density $J^{e x}$ satisfies $\operatorname{supp} J^{e x}(t, \cdot) \cap \Omega=\emptyset$, for any $t>0$ and is assumed to be known.

- $\chi: \mathbb{R} \times \mathbb{R}^{3} \rightarrow \mathbb{R},(t, x) \mapsto \chi(t, x)$, consists in a combination of the electric susceptibility $\xi$ and the conductivity $\sigma$ which may vary according to the medium (this will be specified in the sequel). The exterior part $\chi^{e x}: \mathbb{R} \times \mathbb{R}^{3} \rightarrow \mathbb{R}$ is assumed to be constant (but not necessarily equal to 0 ) with respect to space in the exterior domain, the so-called background medium. Then, we can identify $\Omega$ as the support of $\chi-\chi^{e x}$. This implies that $\hat{\chi}-\hat{\chi}^{e x}$ has (spatial) compact support as well. Here $\hat{\chi}$ denotes the Fourier transform of $\chi$ with respect to time (whose definition is recalled in the sequel in equation (2.2)). In addition, we assume $\chi(t, \cdot)=0$ and $\chi^{e x}(t, \cdot)=0$ for $t<0$.

- $\epsilon_{0}, \mu_{0}$ are the known permittivity and permeability of vacuum, respectively. On can see examples of typical values of all the physical quantities in subsection 2.3;

- $c_{0}=1 / \sqrt{\epsilon_{0} \mu_{0}}$ is the speed of light in vacuum;

- as the medium is non-magnetic, we set the magnetic permeability $\mu$ of the medium to $\mu(t, x)=\mu_{0}$ for every $(t, x) \in \mathbb{R} \times \mathbb{R}^{3}$.

Let us now recall Maxwell's equations. For every $(t, x) \in \mathbb{R} \times \mathbb{R}^{3}$, the electric field $E$, the magnetic field $H$ and the electric displacement $D$ satisfy

$$
\begin{aligned}
\operatorname{curl}_{x} E(t, x) & =-\mu_{0} \frac{\partial H}{\partial t}(t, x), \\
\operatorname{curl}_{x} H(t, x) & =\frac{\partial D}{\partial t}(t, x)+J(t, x) .
\end{aligned}
$$

where we decompose the electric current density $J$ into $J:=J^{i n}+J^{e x}$. The interior part $J^{i n}$ corresponds to the current density generated by ohmic losses while $J^{e x}$ is due to the electromagnetic primary excitation. 
As the medium is considered to be linear, isotropic, inhomogeneous and non-magnetic, we get, for every $(t, x) \in \mathbb{R}_{+} \times \mathbb{R}^{3}$

$$
D(t, x)=\epsilon_{0}\left(E(t, x)+\int_{0}^{\infty} \xi(\tau, x) E(t-\tau, x) d \tau\right)=\epsilon_{0}\left(E+\xi *_{t} E\right)(t, x),
$$

where $*_{t}$ denotes the time convolution operator (indeed $\xi(t, \cdot)=0$ for $t<0$ ). This reads, in the frequency domain,

$$
\hat{D}(\omega, x)=\epsilon_{0}(1+\hat{\xi}(\omega, x)) \hat{E}(\omega, x), \quad(\omega, x) \in \mathbb{R} \times \mathbb{R}^{3}
$$

where $\hat{f}$ stands for the Fourier transform of a function $f$ with respect to $t$

$$
\hat{f}(\omega, x)=\int_{-\infty}^{\infty} f(t, x) e^{i \omega t} d t
$$

and $i^{2}=-1$. Similarly,

$$
J^{i n}(t, x)=\int_{0}^{\infty} \sigma(\tau, x) E(t-\tau, x) d \tau=\left(\sigma *_{t} E\right)(t, x), \quad(t, x) \in \mathbb{R}_{+} \times \mathbb{R}^{3},
$$

or in the frequency domain,

$$
\hat{J}^{i n}(\omega, x)=\hat{\sigma}(\omega, x) \hat{E}(\omega, x), \quad(\omega, x) \in \mathbb{R} \times \mathbb{R}^{3} .
$$

Thus, the exact definition of $\chi$ is given very easily in the frequency domain by

$$
\hat{\chi}(\omega, x)=\hat{\xi}(\omega, x)+\frac{i}{\epsilon_{0} \omega} \hat{\sigma}(\omega, x), \quad(\omega, x) \in \mathbb{R} \backslash\{0\} \times \mathbb{R}^{3} .
$$

Remark 2.1. We do not specify the mathematical framework by now. We assume that $\chi$ belongs to a suitable space that allows to define $\hat{\chi}$. This is the case, for example if $(\xi(\cdot, x), \sigma(\cdot, x)) \in L^{2}(\mathbb{R})$ and $\sigma$ is differentiable (with respect to time).

For any given $\omega$, the electromagnetic fields satisfy for any point $x$ in $\mathbb{R}^{3}$

$$
\begin{aligned}
& \operatorname{curl}_{x} \hat{E}(\omega, x)=i \omega \mu_{0} \hat{H}(\omega, x), \\
& \operatorname{curl}_{x} \hat{H}(\omega, x)=-i \omega \epsilon_{0}(1+\hat{\chi}(\omega, x)) \hat{E}(\omega, x)+\hat{J}^{e x}(\omega, x),
\end{aligned}
$$

that is, for every $(\omega, x) \in \mathbb{R} \times \mathbb{R}^{3}$

$$
\operatorname{curl}_{x} \operatorname{curl}_{x} \hat{E}(\omega, x)-\frac{\omega^{2}}{c_{0}^{2}}(1+\hat{\chi}(\omega, x)) \hat{E}(\omega, x)=i \omega \mu_{0} \hat{J}^{e x}(\omega, x) .
$$

If we assume that the support of all the sources $J^{e x}$ is bounded and that the sources and the inhomogeneities are all located within a finite distance from the origin of the coordinate system, then boundary conditions at infinity are provided by the Silver-Müller radiation condition

$$
\lim _{|x| \rightarrow \infty}\left(x \times \operatorname{curl}_{x} \hat{E}-i \frac{\omega}{c_{0}} \sqrt{1+\hat{\chi}^{e x}}|x| \hat{E}\right)=0,
$$

uniformly in $x /|x|$. Here, $a \times b$ denotes the $\mathbb{R}^{3}$ vectorial product, while $a \cdot b$ denotes the $\mathbb{R}^{3}$ scalar product and $|a|$ the (euclidean) associated norm of vectors $a, b \in \mathbb{R}^{3}$. 


\subsubsection{Heating function}

Let us now focus on the heating function (density of absorbed power $\left(\mathrm{W} / \mathrm{m}^{3}\right)$ ) caused by the electromagnetic source excitation. The total electromagnetic energy density is given by $\mathbf{E}=$ $\frac{1}{2}\left(E \cdot D+\mu_{0} H \cdot H\right)$. By virtue of Maxwell's equations, we get for every $(t, x) \in \mathbb{R}_{+} \times \mathbb{R}^{3}$

$$
-\operatorname{div}_{x} Q(t, x)=\mu_{0}\left(H \cdot \frac{\partial H}{\partial t}\right)(t, x)+\left(E \cdot \frac{\partial D}{\partial t}\right)(t, x)+(E \cdot J)(t, x),
$$

where $Q(t, x)=E(t, x) \times H(t, x)$ is the Poynting vector [18]. Therefore, considering Maxwell's equations, we obtain

$$
-\operatorname{div}_{x} Q(t, x)=\frac{1}{2}\left(E \cdot \frac{\partial D}{\partial t}-\frac{\partial E}{\partial t} \cdot D\right)(t, x)+\frac{\partial \mathbf{E}}{\partial t}(t, x)+(E \cdot J)(t, x) .
$$

The absorbed power out of the electromagnetic source excitation is given by

$$
\Pi(t, x):=\frac{1}{2}\left(E \cdot \frac{\partial D}{\partial t}-\frac{\partial E}{\partial t} \cdot D\right)(t, x)+\left(E \cdot J^{i n}\right)(t, x),
$$

since the term $\frac{\partial \mathbf{E}}{\partial t}$ describes the rate of change of the energy and the term $J^{e x}$ only concerns the external sources.

However, due to the difference between the electromagnetic and thermal time scales, the absorbed density $\Pi_{a}$ has to be computed by the mean of an average on a time interval of critical length $T_{c}$ sufficiently large,

$$
\Pi_{a}(t, x):=\langle\Pi\rangle_{T_{c}}(t, x)=\frac{1}{T_{c}} \int_{t-T_{c}}^{t} \Pi(\tau, x) d \tau .
$$

\subsubsection{Slowly time-varying envelope approximation}

The critical time $T_{c}$ depends on the time scale coupling the different phenomena (electromagnetic, thermal and acoustic). Indeed, the acoustic and the electromagnetic waves have different wave velocities. From a numerical point of view, $T_{c}$ will give the ratio between the time steps of acoustic and electromagnetic computations. This average value can be considered to be equal to the rate of the energy density deposited in the material at position $x$, during the total duration of one period or pseudo period of the illumination process. It can be made precise in the case where the electromagnetic signal presents a slowly time-varying envelope. This assumption makes sense when the high-frequency electromagnetic excitation signal is modulated by a pulse whose temporal width is several decades of times longer than the high-frequency oscillation pe$\operatorname{riod} T_{c}=2 \pi / \omega_{c}$. In that case, the source has a narrow bandwidth in $W_{c}:=\left[\omega_{c}-\Delta_{c}, \omega_{c}+\Delta_{c}\right]$. Such situations occur in microwave setups, which rely on high power pulse-modulated carrier frequency generators, usually operating in the $\mathrm{GHz}$ frequency range, with pulse duration in the $\mu$ s range in order to ensure sufficient energy deposition into the sample, with the drawback of reducing the spatial resolution to the mm range $[9,19,20,24,27,29,35,36]$.

The excitation density current takes the form

$$
J^{e x}(t, x)=\Re\left(j^{e x}(t, x) e^{-i \omega_{c} t}\right),
$$


where $\Re$ stands for the real part and the complex amplitude $j^{e x}$ has a slowly varying envelope on a short period $T_{c}$. Let us recall that if $A(t, x)=\Re\left(a(t, x) e^{-i \omega_{c} t}\right)$ is a signal with a slowly varying envelope then

$$
\left|\frac{\partial^{2} a}{\partial t^{2}}(t, x)\right| \ll \omega_{c}\left|\frac{\partial a}{\partial t}(t, x)\right| \ll \omega_{c}^{2}|a(t, x)|, \quad(t, x) \in \mathbb{R} \times \mathbb{R}^{3}
$$

Note that the above relation comes from

$$
\frac{\partial^{2}\left(a(t, x) e^{-i \omega_{c} t}\right)}{\partial t^{2}}=\left[\frac{\partial^{2} a(t, x)}{\partial t^{2}}-2 i \omega_{c} \frac{\partial a(t, x)}{\partial t}-\omega_{c}^{2} a(t, x)\right] e^{-i \omega_{c} t} .
$$

In practice, the rate of the energy density time-averaged over the short period $T_{c}$ is of interest. Before deriving the form of $\Pi_{a}$ for this specific form of $J^{e x}$, we address the following properties:

- the electromagnetic properties of the medium, given by $\chi$ or equivalently by $\xi$ and $\sigma$, are assumed to be constant with respect to the frequency in the range $W_{c}$ and depend only on the central frequency, namely,

$$
\hat{\xi}(\omega, x) \simeq \hat{\xi}\left(\omega_{c}, x\right), \quad \hat{\sigma}(\omega, x) \simeq \hat{\sigma}\left(\omega_{c}, x\right), \quad \omega \in W_{c} .
$$

- If the current density is given by equation (2.9), then the quantities $E, D$ and $J^{i n}$ admit the same decomposition with slowly varying amplitude:

$$
\begin{aligned}
E(t, x) & =\Re\left(e(t, x) e^{-i \omega_{c} t}\right), & e(t, x) & =\frac{e^{i \omega_{c} t}}{\pi} \int_{0}^{\infty} \hat{E}(\omega, x) e^{-i \omega t} d \omega \\
D(t, x) & =\Re\left(d(t, x) e^{-i \omega_{c} t}\right), & d(t, x) & =\frac{e^{i \omega_{c} t}}{\pi} \int_{0}^{\infty} \hat{D}(\omega, x) e^{-i \omega t} d \omega \\
J^{i n}(t, x) & =\Re\left(j^{i n}(t, x) e^{-i \omega_{c} t}\right), & j^{i n}(t, x) & =\frac{e^{i \omega_{c} t}}{\pi} \int_{0}^{\infty} \hat{J}^{i n}(\omega, x) e^{-i \omega t} d \omega .
\end{aligned}
$$

This follows from the unique solvability of Maxwell's equations and the fact that $\hat{E}, \hat{D}$ and $\hat{J}^{i n}$ are also supported in $W_{c}$. In particular,

$$
d(t, x) \simeq \epsilon_{0}\left(1+\hat{\xi}\left(\omega_{c}, x\right)\right) e(t, x) .
$$

This comes from

$$
\begin{aligned}
d(t, x) e^{-i \omega_{c} t} & =\frac{1}{\pi} \int_{0}^{\infty} \hat{D}(\omega, x) e^{-i \omega t} d \omega \\
& =\frac{1}{\pi} \int_{W_{c}} \epsilon_{0}(1+\hat{\xi}(\omega, x)) \hat{E}(\omega, x) e^{-i \omega t} d \omega \\
& \simeq \frac{\epsilon_{0}}{\pi}\left(1+\hat{\xi}\left(\omega_{c}, x\right)\right) \int_{W_{c}} \hat{E}(\omega, x) e^{-i \omega t} d \omega \\
& =\epsilon_{0}\left(1+\hat{\xi}\left(\omega_{c}, x\right)\right) e(t, x) e^{-i \omega_{c} t}
\end{aligned}
$$

Similarly,

$$
j^{i n}(t, x) \simeq \hat{\sigma}\left(\omega_{c}, x\right) e(t, x)
$$


- Let $A(t, x)=\Re\left(a(t, x) e^{-i \omega_{c} t}\right)$ and $B(t, x)=\Re\left(b(t, x) e^{-i \omega_{c} t}\right)$ be two slowly varying envelope signals. We obtain for the averaged value over $T_{c}$ for the product of these two functions

$$
\langle A \cdot B\rangle_{T_{c}}(t, x) \simeq \frac{1}{2} \Re(a(t, x) \cdot \overline{b(t, x)}),
$$

since

$$
\begin{aligned}
A \cdot B & =\frac{1}{4}\left(a e^{-i \omega_{c} t}+\overline{a e^{-i \omega_{c} t}}\right) \cdot\left(b e^{-i \omega_{c} t}+\overline{b e^{-i \omega_{c} t}}\right) \\
& =\frac{1}{4}\left(a \cdot b e^{-i 2 \omega_{c} t}+\overline{a \cdot b} e^{i 2 \omega_{c} t}\right)+\frac{1}{4}(a \cdot \bar{b}+\bar{a} \cdot b) .
\end{aligned}
$$

Here $\bar{a}$ stands for the complex conjugate of $a$. The first two terms correspond to a signal with high frequency components while the last two terms are slowly varying with respect to $t$. Thus, their short-time average value is given by

$$
\langle A \cdot B\rangle_{T_{c}}(t, x) \simeq \frac{1}{4}\langle a \cdot \bar{b}+\bar{a} \cdot b\rangle_{T_{c}}(t, x),
$$

resulting in (2.11). One can also show that $\left\langle A \cdot \frac{\partial A}{\partial t}\right\rangle_{T_{c}}(t, x) \simeq 0$.

Therefore, the short-time averaged absorbed energy $\Pi_{a}$ defined in equation (2.8), using (2.4), (2.11) and the above assumptions, is given by

$$
\Pi_{a}(t, x) \simeq \frac{\epsilon_{0} \omega_{c}}{2} \Im\left(\hat{\chi}\left(\omega_{c}, x\right)\right)|e(t, x)|^{2}=\frac{\epsilon_{0} \omega_{c}}{2 \pi^{2}} \Im\left(\hat{\chi}\left(\omega_{c}, x\right)\right)\left|\int_{0}^{\infty} \hat{E}(\omega, x) e^{-i \omega t} d \omega\right|^{2} .
$$

where $\Im(a)$ stands for the imaginary part of the complex number $a$.

\subsection{Pressure and temperature equations}

Once we have estimated the absorbed energy $\Pi_{a}$, we may focus on the heating/pressure wave generation processes. Temperature rise and pressure are coupled. Note that most of the TAT models of the literature do not consider the heating process, which is often assumed to be instantaneous. This approximation can be justified in the photoacoustic case, where time scales are quite different: roughly speaking the lightning (and heating) process is much faster than the acoustic wave propagation. When the source is a radio-frequency one, this assumption may not be relevant any longer.

According to [10], the temperature and the pressure are determined by coupled equations which derive from the mass, momentum and energy conservation equations. Assuming that the variations in temperature and pressure are weak, the nonlinear effects are neglected and we obtain the following set of equations, for the temperature rise $\theta$ and the pressure perturbation $p$ from the equilibrium steady state:

$$
\left\{\begin{aligned}
\frac{\partial^{2}}{\partial t^{2}}\left(K_{\theta} p-\beta \theta\right)(t, x) & =\operatorname{div}_{x}\left(\frac{1}{\rho} \nabla p\right)(t, x), & & (t, x) \in \mathbb{R}_{+} \times \mathbb{R}^{3}, \\
\frac{\partial}{\partial t}\left(\rho C_{p} \theta-\theta_{0} \beta p\right)(t, x) & =\operatorname{div}_{x}(\kappa \nabla \theta)(t, x)+\Pi_{a}(t, x), & & (t, x) \in \mathbb{R}_{+} \times \mathbb{R}^{3},
\end{aligned}\right.
$$

where 
- $K_{\theta}$ is the isothermal compressibility,

- $\beta$ is the volume thermal expansivity,

- $\rho$ is the mass density at steady state,

- $\kappa$ is the thermal conductivity,

- $\theta_{0}$ is the background temperature,

- and $C_{p}$ is the isobar specific heat capacity (quantity of heat required to raise the temperature of a unit mass).

All these parameter are assumed to be independent of the time $t$, but may depend on the position $x$, see Table 1 for some typical values of these parameters.

A combination of the above equations leads to

$$
\left(\rho K_{\theta}-\frac{\theta_{0} \beta^{2}}{C_{p}}\right) \frac{\partial^{2} p}{\partial t^{2}}-\rho \operatorname{div}_{x}\left(\frac{1}{\rho} \nabla p\right)=\frac{\beta}{C_{p}} \frac{\partial \Pi_{a}}{\partial t}+\frac{\beta}{C_{p}} \frac{\partial}{\partial t} \operatorname{div}_{x}(\kappa \nabla \theta) .
$$

Let us introduce the acoustic wave velocity $v_{s}$ which is defined by

$$
\frac{1}{v_{s}^{2}}=\rho K_{\theta}-\frac{\theta_{0} \beta^{2}}{C_{p}}
$$

to obtain the thermoacoustic equation in heterogeneous media

$$
\frac{1}{v_{s}^{2}} \frac{\partial^{2} p}{\partial t^{2}}-\rho \operatorname{div}_{x}\left(\frac{1}{\rho} \nabla p\right)=\frac{\beta}{C_{p}} \frac{\partial \Pi_{a}}{\partial t}+\frac{\beta}{C_{p}} \frac{\partial}{\partial t} \operatorname{div}_{x}(\kappa \nabla \theta) .
$$

We further introduce the Grüneisen parameter $\Gamma$ defined as

$$
\Gamma=\frac{\beta v_{s}^{2}}{C_{p}}
$$

It is worth mentioning that $v_{s}$ and the Grüneisen parameter $\Gamma$ may be spatially heterogeneous. The coupled equations now read

$$
\left\{\begin{aligned}
& \frac{\partial^{2} p}{\partial t^{2}}-\rho v_{s}^{2} \operatorname{div}_{x}\left(\frac{1}{\rho} \nabla p\right)-\Gamma \frac{\partial}{\partial t} \operatorname{div}_{x}(\kappa \nabla \theta)=\Gamma \frac{\partial \Pi_{a}}{\partial t}, \quad(t, x) \in \mathbb{R}_{+} \times \mathbb{R}^{3}, \\
& \frac{\partial \theta}{\partial t}-\frac{1}{\rho C_{p}} \operatorname{div}_{x}(\kappa \nabla \theta)-\frac{\theta_{0} \beta}{\rho C_{p}} \frac{\partial p}{\partial t}=\frac{\Pi_{a}}{\rho C_{p}}, \quad(t, x) \in \mathbb{R}_{+} \times \mathbb{R}^{3} .
\end{aligned}\right.
$$

The initial conditions are such that there are no temperature or pressure differences before the absorption of the electromagnetic energy:

$$
\theta(0, x)=0, \quad p(0, x)=0, \quad \frac{\partial p}{\partial t}(0, x)=0, \quad x \in \mathbb{R}^{3} .
$$

Without loss of generality, we reformulate equation (2.15) by introducing

$$
P(t, x)=\int_{0}^{t} p(\tau, x) d \tau
$$


which corresponds to the integrated pressure. As $\frac{\partial P}{\partial t}=p$, integrating the first equation in (2.15) with respect to $t$ gives

$$
\left\{\begin{array}{r}
\frac{\partial^{2} P}{\partial t^{2}}-\rho v_{s}^{2} \operatorname{div}_{x}\left(\frac{1}{\rho} \nabla P\right)-\Gamma \operatorname{div}_{x}(\kappa \nabla \theta)=\Gamma \Pi_{a}, \quad(t, x) \in \mathbb{R}_{+} \times \mathbb{R}^{3}, \\
\frac{\partial \theta}{\partial t}-\frac{1}{\rho C_{p}} \operatorname{div}_{x}(\kappa \nabla \theta)-\frac{\theta_{0} \beta}{\rho C_{p}} \frac{\partial^{2} P}{\partial t^{2}}=\frac{\Pi_{a}}{\rho C_{p}}, \quad(t, x) \in \mathbb{R}_{+} \times \mathbb{R}^{3} .
\end{array}\right.
$$

with the initial conditions

$$
\theta(0, x)=0, \quad P(0, x)=0, \quad \frac{\partial P}{\partial t}(0, x)=0, \quad x \in \mathbb{R}^{3} .
$$

\subsection{Typical values of soft tissues}

To be able to fully understand the time scale and evolution of the various physical quantities, some typical values of the various physical parameters are provided in Table 1 . These typical values correspond to the ones of soft tissues that can be found when one considers a biomedical application, as for example breast cancer imaging [8, 11, 14, 23].

\begin{tabular}{|l|c||c|c|}
\hline Parameter & Symbol & Value & Units \\
\hline Permittivity of the vacuum & $\epsilon_{0}$ & $8.8510^{-12}$ & $\mathrm{~F} . \mathrm{m}^{-1}$ \\
Permeability of the vacuum & $\mu_{0}$ & $1.2510^{-6}$ & $\mathrm{H} . \mathrm{m}^{-1}$ \\
Electric susceptibility (fat/glandular) & $\xi$ & $7.3 / 35.5$ & - \\
Conductivity (fat/glandular) & $\sigma$ & $0.23 / 1.34$ & $\mathrm{~S} . \mathrm{m}^{-1}$ \\
Isobar specific heat capacity & $C_{p}$ & $3.5-4 \times 10^{3}$ & $\mathrm{~J} . \mathrm{K}^{-1} \cdot \mathrm{kg}^{-1}$ \\
Isothermal compressibility & $K_{\theta} \sim \frac{1}{\rho v_{s}^{2}}$ & $5 \times 10^{-10}$ & $\mathrm{~Pa}^{-1}$ \\
Volume thermal expansivity & $\beta$ & $4 \times 10^{-4}$ & $\mathrm{~K}^{-1}$ \\
Mass density & $\rho$ & 1000 & $\mathrm{~kg} \cdot \mathrm{m}^{-3}$ \\
Thermal conductivity & $\kappa$ & 0.4 & $\mathrm{~W} . \mathrm{m}^{-1} \cdot \mathrm{K}^{-1}$ \\
Background temperature $\left(20^{\circ} \mathrm{C}\right)$ & $\theta_{0}$ & 293 & $\mathrm{~K}$ \\
\hline
\end{tabular}

Table 1: Approximated values of the physical parameters. The electric susceptibility and conductivity are measured at $3 \mathrm{GHz}$ and at room temperature and correspond to fat and glandular tissues, respectively.

The acoustic transducers are typically wide band sensors with a central frequency of the order of 1 to $10 \mathrm{MHz}$, leading to an acoustic wavelength $\lambda_{a}$ in the range of 0.1 to $1 \mathrm{~mm}[9,27]$. Meanwhile, the thermal diffusivity $D_{\theta}=\kappa /\left(\rho C_{p}\right)$ is approximately equal to $10^{-7} \mathrm{~m}^{2} \mathrm{~s}^{-1}$. Hence, the thermal confinement threshold $\left(\lambda_{a} / 2\right)^{2} / D_{\theta}$ is of the order of 0.05 to $5 \mathrm{~s}$ [34]. For highresolution imaging, the thermal confinement duration is more or less 10 times the duration of the illumination (which lasts few $\mu \mathrm{s}$ ). Thus, in those cases, it seems rather difficult to neglect at first glance the coupling term between the thermal and acoustic effects. 


\section{Modelling of the inverse problem within the slowly time- varying envelope approximation}

\subsection{The infinite dimensional model}

As we have already seen, if the electromagnetic signal presents a slowly time-varying envelope, meaning that $J^{e x}$ takes the form (2.9), then $\hat{E}$ and $\hat{\chi}$ admit frequency support in $W_{c}=\left[\omega_{c}-\right.$ $\left.\Delta_{c}, \omega_{c}+\Delta_{c}\right]$ and

$$
\hat{\chi}(\omega, x) \simeq \hat{\chi}\left(\omega_{c}, x\right) \text { for all } x \in \mathbb{R}^{3}, \omega \in W_{c} .
$$

In the sequel, we denote $\hat{\chi}_{c}:=\hat{\chi}\left(\omega_{c}, \cdot\right)$ and $\hat{\chi}_{c}^{e x}:=\hat{\chi}^{e x}\left(\omega_{c}, \cdot\right)$. We recall that $\hat{\chi}_{c}-\hat{\chi}_{c}^{e x}$ has (spatial) compact support.

So, putting together the equations (2.5), (2.12) and (2.16), the direct problem reads: Solve the system

$$
\left\{\begin{array}{r}
\operatorname{curl}_{x} \operatorname{curl}_{x} \hat{E}-\frac{\omega^{2}}{c_{0}^{2}}\left(1+\hat{\chi}_{c}\right) \hat{E}=i \omega \mu_{0} \hat{J}^{e x} \text { in } W_{c} \times \mathbb{R}^{3} \\
\Pi_{a}(t, x)=\frac{\epsilon_{0} \omega_{c}}{2 \pi^{2}} \Im\left(\hat{\chi}_{c}\right)(x)\left|\int_{W_{c}} \hat{E}(\omega, x) e^{-i \omega t} d \omega\right|^{2} \text { in } \mathbb{R}_{+} \times \mathbb{R}^{3} \\
\frac{\partial^{2} P}{\partial t^{2}}-\rho v_{s}^{2} \operatorname{div}_{x}\left(\frac{1}{\rho} \nabla P\right)-\Gamma \operatorname{div}_{x}(\kappa \nabla \theta)=\Gamma \Pi_{a} \text { in } \mathbb{R}_{+} \times \mathbb{R}^{3}, \\
\frac{\partial \theta}{\partial t}-\frac{1}{\rho C_{p}} \operatorname{div}_{x}(\kappa \nabla \theta)-\frac{\theta_{0} \beta}{\rho C_{p}} \frac{\partial^{2} P}{\partial t^{2}}=\frac{\Pi_{a}}{\rho C_{p}} \text { in } \mathbb{R}_{+} \times \mathbb{R}^{3},
\end{array}\right.
$$

with initial conditions

$$
\theta(0, x)=0, P(0, x)=\frac{\partial P}{\partial t}(0, x)=0 \quad \text { for all } x \in \mathbb{R}^{3},
$$

together with the radiation condition

$$
\lim _{|x| \rightarrow \infty}\left(x \times \operatorname{curl}_{x} \hat{E}-i \frac{\omega}{c_{0}} \sqrt{1+\hat{\chi}^{e x}}|x| \hat{E}\right)=0,
$$

where the convergence should be uniformly in $\frac{x}{|x|}$, see (2.6); to obtain the Fourier transform of the electric field $\hat{E}$, the averaged absorbed energy $\Pi_{\alpha}$, the temperature rise $\theta$ (supported in $\Omega$ ) and the integrated pressure $P$.

Remark 3.1. - There exist partial results for Maxwell's equations (3.1a). We know, for example that for a fixed frequency $\omega_{c}$, if $\hat{\chi}_{c} \in L^{\infty}\left(\mathbb{C}^{3}\right)$ and $\hat{J}_{c}^{e x} \in L^{2}\left(\mathbb{C}^{3}\right)$ are compact supported (up to a constant function) in $\mathbb{R}^{3}$, then the problem (3.1a) has a unique solution in $H_{\text {loc }}\left(\operatorname{curl}_{x}, \mathbb{R}^{3}\right)$, see [25], where

$$
H_{\text {loc }}\left(\operatorname{curl}_{x}, \mathbb{R}^{3}\right)=\left\{V: \mathbb{R}^{3} \rightarrow \mathbb{C}^{3}:\left.V\right|_{B} \in H\left(\operatorname{curl}_{x}, B\right) \text { for all balls } B \subseteq \mathbb{R}^{3}\right\}
$$

and $H\left(\operatorname{curl}_{x}, B\right)=\left\{V \in L^{2}(B) \mid \operatorname{curl}_{x} V \in L^{2}(B)\right\}$.

- There are no straightforward existence and/or uniqueness results for the coupled system (3.1c) (3.1d). If we fix one of the variables $\theta$ or $P$, the equations are classical and we get many existence and regularity results under assumptions on the fixed unknown. However, the coupling imposes to use specific methods (fixed point or diagonalization methods) that we don't want to check in the present paper. This will be addressed in a forthcoming work where rigorous mathematical analysis will be performed. 
Remark 3.2. In the above derivation, we considered the total (electromagnetic) field. We didn't use the perturbed field (or scattered field) $E_{s}$ which is directly linked to $\hat{\chi}-\hat{\chi}^{\text {ex }}$ since the dissipation $\Pi_{a}$ as a function of $E_{s}$ is much more complicated.

We are now interested in the inverse problem: given a known source $J^{e x}$ and integrated pressure measurements $P_{\text {obs }}$ on a set $(0, T) \times \mathcal{W}$, recover $\hat{\chi}_{c}$. Here, the set $\mathcal{W}$ denotes the area of the transducers. Following [7] we define the functional $\mathcal{J}$ by

$$
\mathcal{J}\left(\hat{\chi}_{c}\right)=\mathcal{F}\left(\hat{\chi}_{c}\right)+f\left(\hat{\chi}_{c}\right) .
$$

Here $f\left(\hat{\chi}_{c}\right)$ stands for a regularizing term and $\mathcal{F}$ is a least square functional with respect to the measured integrated pressure data. We set

$$
\mathcal{F}\left(\hat{\chi}_{c}\right)=\frac{1}{2} \int_{(0, T) \times \mathcal{W}}\left(P\left[\hat{\chi}_{c}\right](t, x)-P_{o b s}(t, x)\right)^{2} \mathrm{~d} x \mathrm{~d} t
$$

where $P_{\text {obs }}$ is the measured pressure on $(0, T) \times \mathcal{W}$ when the source signal is $J^{e x}$. Here $P\left[\hat{\chi}_{c}\right]$ is the solution of equations (3.1c) - (3.1d) with (3.2). We may define $f$ as

$$
f\left(\hat{\chi}_{c}\right)=\frac{\alpha_{0}}{2} \int_{W_{c} \times \mathbb{R}^{3}}\left|\hat{\chi}-\hat{\chi}^{e x}\right|^{2}(\omega, x) d \omega d x=\frac{2 \Delta_{c} \alpha_{0}}{2} \int_{\mathbb{R}^{3}}\left|\hat{\chi}_{c}-\hat{\chi}_{c}^{e x}\right|^{2}(x) d x
$$

where $\alpha_{0}>0$, for example. Then, the minimization problem reads

$$
\min _{\hat{\chi}_{c} \in \mathcal{U}_{a d}} \mathcal{J}\left(\hat{\chi}_{c}\right)
$$

where $\mathcal{U}_{a d}$ is a closed set of admissible functions $\hat{\chi}_{c}$ such that $\hat{\chi}_{c}=\hat{\chi}_{c}^{e x}$ outside $\Omega$.

In the sequel, we set $\alpha=2 \Delta_{c} \alpha_{0}$ so that

$$
f\left(\hat{\chi}_{c}\right)=\frac{\alpha}{2} \int_{\mathbb{R}^{3}}\left|\hat{\chi}_{c}-\hat{\chi}_{c}^{e x}\right|^{2}(x) d x .
$$

\subsection{Formal formulation of the inverse problem}

The above formulation is of course quite formal. The study of problem (3.5) in an infinite dimensional setting is challenging and deserves to be precisely studied. Indeed, the operator $\mathcal{T}: \hat{\chi}_{c} \mapsto P\left[\hat{\chi}_{c}\right]$ is not easy to handle. In particular, its continuity properties strongly depend on the functional framework and regularity properties of the solutions of the strongly coupled system (3.1).

We now give a formal analysis of the inverse problem to present the whole reconstruction process. This may be completely justified in a finite dimensional (discrete) setting. It is not our concern to enter in the discretization details and we keep the same notations as in the infinite dimensional setting. However, as we have numerics in mind, we have replaced $\mathbb{R}_{+}$by $(0, T)$ with some terminal time $T$ large enough, and $\mathbb{R}^{3}$ by some large ball $\mathcal{B}$. We use a Dirichlet boundary condition on $\partial \mathcal{B}$ (recall that $T$ is chosen large enough to allow it). The discretization process is a challenging issue that we don't address in the present paper. 
The direct problem now reads

$$
\left\{\begin{array}{c}
\operatorname{curl}_{x} \operatorname{curl}_{x} \hat{E}-\frac{\omega^{2}}{c_{0}^{2}}\left(1+\hat{\chi}_{c}\right) \hat{E}=i \omega \mu_{0} \hat{J}^{e x} \text { in } W_{c} \times \mathcal{B} \\
\Pi_{a}(t, x)=\frac{\epsilon_{0} \omega_{c}}{2 \pi^{2}} \Im\left(\hat{\chi}_{c}(x)\right)\left|\int_{W_{c}} \hat{E}(\omega, x) e^{-i \omega t} d \omega\right|^{2} \text { in }(0, T) \times \mathcal{B} \\
\frac{\partial^{2} P}{\partial t^{2}}-\rho v_{s}^{2} \operatorname{div}_{x}\left(\frac{1}{\rho} \nabla P\right)-\Gamma \operatorname{div}_{x}(\kappa \nabla \theta)=\Gamma \Pi_{a}, \text { in }(0, T) \times \mathcal{B} \\
\frac{\partial \theta}{\partial t}-\frac{1}{\rho C_{p}} \operatorname{div}_{x}(\kappa \nabla \theta)-\frac{\theta_{0} \beta}{\rho C_{p}} \frac{\partial^{2} P}{\partial t^{2}}=\frac{\Pi_{a}}{\rho C_{p}}, \text { in }(0, T) \times \mathcal{B}
\end{array}\right.
$$

with initial conditions

$$
\theta(0, x)=0, \quad P(0, x)=\frac{\partial P}{\partial t}(0, x)=0 \text { in } \mathcal{B},
$$

and boundary conditions

$$
\begin{array}{r}
\hat{E}(\omega, x)=0, \quad(\omega, x) \in W_{c} \times \partial \mathcal{B} \\
P(t, x)=0, \quad \frac{\partial P}{\partial t}(t, x)=0, \quad \theta(t, x)=0, \quad(t, x) \in(0, T) \times \partial \mathcal{B} .
\end{array}
$$

Remark 3.3. We decided to set a Dirichlet boundary condition for the $\hat{E}$ field. This is the case for example if either

- $\hat{\chi}^{\text {ex }}$ is lossless and the ball $\mathcal{B}$ is very large,

- $\hat{\chi}^{e x}$ is lossless and there is a metallic casing around $\mathcal{B}$, or

- $\hat{\chi}^{e x}$ is lossy and the waves will be automatically damped.

Another possibility could be to use a Robin type boundary, which can be seen as a first-order approximation of the Silver-Müller radiation boundary condition on a bounded surface:

$$
n \times \operatorname{curl}_{x} \hat{E}-i \frac{\omega}{c_{0}} \sqrt{1+\hat{\chi}^{e x}} n \times n \times \hat{E}=0,
$$

where $n$ is the unit outward normal vector on $\partial \mathcal{B}$.

Note that, the issue is the same for the acoustic pressure, but as we are looking for $p$ (or P) which only correspond to the variation of the pressure with respect to the initial one, it seems rather natural to assume that $P=0$ on the boundary.

Theorem 3.1. Assume $\mathcal{U}_{a d}$ is bounded or $\alpha>0$. Then, the discrete minimization problem has at least a solution.

Proof. This is clear since either the admissible set $\mathcal{U}_{a d}$ is bounded or $\mathcal{J}$ is coercive. Moreover, the continuity properties are fulfilled in the discrete framework.

Uniqueness is a challenging problem, since the cost functional is not convex (because of the fitting data term). Nevertheless, any solution $\hat{\chi}_{c}^{*}$ satisfies

$$
\nabla \mathcal{J}\left(\hat{\chi}_{c}^{*}\right) \cdot\left(\hat{\chi}_{c}-\hat{\chi}_{c}^{*}\right) \geq 0 \text { for all } \hat{\chi}_{c} \in \mathcal{U}_{a d}
$$


Next subsections are devoted to the computation of $\nabla \mathcal{J}\left(\hat{\chi}_{c}^{*}\right)$ and the setting of a necessary optimality system.

We first start to compute $\nabla \mathcal{F}\left(\hat{\chi}_{c}^{*}\right) \cdot \hat{\chi}_{c}$ before tackling the term in $\nabla f\left(\hat{\chi}_{c}^{*}\right) \cdot \hat{\chi}_{c}$. In the sequel $\hat{\chi}_{c}^{*}$ denotes an optimal solution and $\hat{E}^{*}, P^{*}$ and $\theta^{*}$ are the corresponding solutions of (the discrete) system (3.7). We previously adopted the notation $\cdot$ for the $\mathbb{R}^{3}$ inner product. However, in the sequel we omit this dot, to make computations more readable. More precisely, if $A=\left(A_{k}\right)_{1 \leq k \leq 3}$ and $B=\left(B_{k}\right)_{1 \leq k \leq 3}$ are vector valued functions from $\mathbb{R}^{3}$ to $\mathbb{R}^{3}$ we write $A(x) B(x)$ instead of $A(x) \cdot B(x)\left(=\sum_{k=1}^{3} A_{k}(x) B_{k}(x)\right)$.

\subsection{The (discrete) pressure-temperature system}

The coupling between equations $(3.7 \mathrm{a})-(3.7 \mathrm{~b})$ and $(3.7 \mathrm{c})-(3.7 \mathrm{~d})$ is done via the $\Pi_{a}$ term. Let $\hat{\chi}_{c} \in \mathcal{U}_{a d}$ and denote by $\dot{\Pi}_{a}$ the derivative of $\Pi_{a}$ at $\hat{\chi}_{c}^{*}$ in the direction $\hat{\chi}_{c}$ :

$$
\dot{\Pi}_{a} \cdot \hat{\chi}_{c}:=\lim _{\rho \rightarrow 0^{+}} \frac{\Pi_{a}\left(\hat{\chi}_{c}^{*}+\rho \hat{\chi}_{c}\right)-\Pi_{a}\left(\hat{\chi}_{c}^{*}\right)}{\rho} .
$$

Let us denote similarly $\dot{P}, \dot{\theta}$ the derivatives of $P$ and $\theta$ at $\hat{\chi}_{c}^{*}$ in the direction $\hat{\chi}_{c}$. So

$$
\nabla \mathcal{F}\left(\hat{\chi}_{c}^{*}\right) \cdot \hat{\chi}_{c}=\int_{(0, T) \times \mathcal{W}}\left(P^{*}-P_{o b s}\right) \dot{P} d t d x
$$

where $P^{*}=P\left[\hat{\chi}_{c}^{*}\right]$ and $\theta^{*}=\theta\left[\hat{\chi}_{c}^{*}\right]$. The equations satisfied by $\dot{P}$ and $\dot{\theta}$ are

$$
\left\{\begin{aligned}
& \frac{\partial^{2} \dot{P}}{\partial t^{2}}-\rho v_{s}^{2} \operatorname{div}_{x}\left(\frac{1}{\rho} \nabla \dot{P}\right)-\Gamma \operatorname{div}_{x}(\kappa \nabla \dot{\theta})=\Gamma \dot{\Pi}_{a} \text { in }(0, T) \times \mathcal{B}, \\
& \rho C_{p} \frac{\partial \dot{\theta}}{\partial t}-\operatorname{div}_{x}(\kappa \nabla \dot{\theta})-\theta_{0} \beta \frac{\partial^{2} \dot{P}}{\partial t^{2}}=\dot{\Pi}_{a} \quad \text { in }(0, T) \times \mathcal{B},
\end{aligned}\right.
$$

with initial and boundary conditions

$$
\left\{\begin{array}{c}
\dot{\theta}(0, x)=0, \dot{P}(0, x)=0, \quad \frac{\partial \dot{P}}{\partial t}(0, x)=0, \quad x \in \mathcal{B} \\
\dot{P}(t, x)=0, \quad \frac{\partial \dot{P}}{\partial t}(t, x)=0, \quad \dot{\theta}(t, x)=0, \quad(t, x) \in(0, T) \times \partial \mathcal{B} .
\end{array}\right.
$$

Let us define the adjoint system for $\left(P^{*}, \theta^{*}\right)$. We consider the following system for $q^{*}$ and $\zeta^{*}$ :

$$
\left\{\begin{array}{r}
\frac{\partial^{2} q^{*}}{\partial t^{2}}-\theta_{0} \beta \frac{\partial^{2} \zeta^{*}}{\partial t^{2}}-\operatorname{div}_{x}\left(\frac{1}{\rho} \nabla\left(\rho v_{s}^{2} q^{*}\right)\right)=\left(P^{*}-P_{o b s}\right) \mathbf{1}_{(0, T) \times \mathcal{W}} \text { in }(0, T) \times \mathcal{B} \\
-\rho C_{p} \frac{\partial \zeta^{*}}{\partial t}-\operatorname{div}_{x}\left(\kappa \nabla\left(\Gamma q^{*}\right)\right)-\operatorname{div}_{x}\left(\kappa \nabla \zeta^{*}\right)=0 \text { in }(0, T) \times \mathcal{B},
\end{array}\right.
$$

with initial and boundary conditions

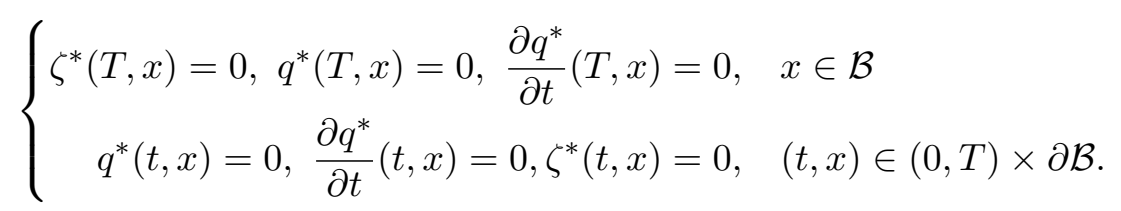

Then we get 
Lemma 3.1. The gradient of $\mathcal{F}$ at $\hat{\chi}_{c}^{*}$ in the direction $\hat{\chi}_{c}$ is given by

$$
\nabla \mathcal{F}\left(\hat{\chi}_{c}^{*}\right) \cdot \hat{\chi}_{c}=\int_{(0, T) \times \mathcal{B}}\left(\Gamma q^{*}+\zeta^{*}\right) \dot{\Pi}_{a} d t d x
$$

where $\left(q^{*}, \zeta^{*}\right)$ are defined by the adjoint equations (3.11)-(3.12), and $\dot{\Pi}_{a}$ is the derivative of $\Pi_{a}$ at $\hat{\chi}_{c}^{*}$ in the direction $\hat{\chi}_{c}$.

Proof. Using integration by parts, we obtain

$$
\begin{aligned}
\nabla \mathcal{F}\left(\hat{\chi}_{c}^{*}\right) \cdot \hat{\chi}_{c}= & \int_{(0, T) \times \mathcal{W}}\left(P^{*}-P_{o b s}\right) \dot{P} d t d x=\int_{(0, T) \times \mathcal{B}}\left(P^{*}-P^{o b s}\right) \dot{P} \mathbf{1}_{(0, T) \times \mathcal{W}} d t d x \\
= & \int_{(0, T) \times \mathcal{B}}\left[\frac{\partial^{2} q^{*}}{\partial t^{2}}-\theta_{0} \beta \frac{\partial^{2} \zeta^{*}}{\partial t^{2}}-\operatorname{div}_{x}\left(\frac{1}{\rho} \nabla\left(\rho v_{s}^{2} q^{*}\right)\right)\right] \dot{P} d t d x \\
& +\underbrace{\int_{(0, T) \times \mathcal{B}}\left(-\rho C_{p} \frac{\partial \zeta^{*}}{\partial t}-\operatorname{div}_{x}\left(\kappa \nabla\left(\Gamma q^{*}\right)\right)-\operatorname{div}_{x}\left(\kappa \nabla \zeta^{*}\right)\right) \dot{\theta} d t d x}_{=0} \\
= & \int_{(0, T) \times \mathcal{B}}\left\{\left[\frac{\partial^{2} \dot{P}}{\partial t^{2}}-\rho v_{s}^{2} \operatorname{div}_{x}\left(\frac{1}{\rho} \nabla \dot{P}\right)\right] q^{*}-\theta_{0} \beta \frac{\partial^{2} \dot{P}}{\partial t^{2}} \zeta^{*}\right\} d t d x \\
& +\int_{(0, T) \times \mathcal{B}}\left\{\left(\rho C_{p} \frac{\partial \dot{\theta}}{\partial t}-\operatorname{div}_{x}(\kappa \nabla \dot{\theta})\right) \zeta^{*}-\Gamma \operatorname{div}_{x}(\kappa \nabla \dot{\theta}) q^{*}\right\} d t d x \\
= & \int_{(0, T) \times \mathcal{B}}\left[\frac{\partial^{2} \dot{P}}{\partial t^{2}}-\rho v_{s}^{2} \operatorname{div}_{x}\left(\frac{1}{\rho} \nabla \dot{P}\right)-\Gamma \operatorname{div}_{x}(\kappa \nabla \dot{\theta})\right] q^{*} d t d x \\
& +\int_{(0, T) \times \mathcal{B}}\left(\rho C_{p} \frac{\partial \dot{\theta}}{\partial t}-\operatorname{div}_{x}(\kappa \nabla \dot{\theta})-\theta_{0} \beta \frac{\partial^{2} \dot{P}}{\partial t^{2}}\right) \zeta^{*} d t d x \\
= & \int_{(0, T) \times \mathcal{B}}\left(\Gamma q^{*}+\zeta^{*}\right) \dot{\Pi}_{a} d t d x .
\end{aligned}
$$

Now, we have to estimate $\dot{\Pi}_{a}$ introducing an adjoint equation for the electromagnetic part.

\section{4 (Discrete) Maxwell's equations}

Let us now define the adjoint state $\Phi^{*}$ associated to $E^{*}$. To simplify the notation, we introduce the quantities

$$
\mathcal{E}^{*}(t, x)=\int_{W_{c}} \hat{E}^{*}(\omega, x) e^{-i \omega t} d \omega \quad \text { and } \quad \dot{\mathcal{E}}(t, x)=\int_{W_{c}} \dot{\hat{E}}(\omega, x) e^{-i \omega t} d \omega .
$$

Then, we set $\Phi^{*}$ to be the solution of the equation

$\operatorname{curl}_{x} \operatorname{curl}_{x} \Phi^{*}(\omega, \cdot)-\frac{\omega^{2}}{c_{0}^{2}}\left(1+\hat{\chi}_{c}^{*}\right) \Phi^{*}(\omega, \cdot)=\Im\left(\hat{\chi}_{c}^{*}\right)\left(\int_{0}^{T}\left(\Gamma q^{*}+\zeta^{*}\right)(t, \cdot) \overline{\mathcal{E}^{*}}(t, \cdot) e^{-i \omega t} d t\right)$ in $W_{c} \times \mathcal{B}$

with the homogeneous Dirichlet boundary conditions

$$
\Phi^{*}(\omega, x)=0, \quad(\omega, x) \in W_{c} \times \partial \mathcal{B} .
$$


Proposition 3.1. The gradient of $\mathcal{F}$ at $\hat{\chi}_{c}^{*}$ in the direction $\hat{\chi}_{c}$ is given by

$$
\begin{aligned}
\nabla \mathcal{F}\left(\hat{\chi}_{c}^{*}\right) \cdot \hat{\chi}_{c}= & \frac{\epsilon_{0} \omega_{c}}{2 \pi^{2}} \int_{\mathcal{B}}\left\{\left(\int_{0}^{T}\left(\Gamma q^{*}+\zeta^{*}\right)(t, x)\left|\mathcal{E}^{*}(t, x)\right|^{2} d t\right) \Im\left(\hat{\chi}_{c}(x)\right)\right. \\
& +2\left(\Re \int_{W_{c}} \frac{\omega^{2}}{c_{0}^{2}} \hat{E}^{*}(\omega, x) \Phi^{*}(\omega, x) d \omega\right) \Re\left(\hat{\chi}_{c}(x)\right) \\
& \left.-2\left(\Im \int_{W_{c}} \frac{\omega^{2}}{c_{0}^{2}} \hat{E}^{*}(\omega, x) \Phi^{*}(\omega, x) d \omega\right) \Im\left(\hat{\chi}_{c}(x)\right)\right\} d x
\end{aligned}
$$

where $\left(q^{*}, \zeta^{*}\right)$ are defined by the adjoint equations (3.11) - (3.12), and $\Phi^{*}$ by (3.14).

Proof. Let us denote by $\dot{\hat{E}}$ the derivative of $\hat{E}$ at $\hat{\chi}_{c}^{*}$ in the direction $\hat{\chi}_{c}$. The equations satisfied by $\dot{\Pi}_{a}$ and $\dot{\hat{E}}$ are

$$
\left\{\begin{array}{c}
\operatorname{curl}_{x} \operatorname{curl}_{x} \dot{\hat{E}}-\frac{\omega^{2}}{c_{0}^{2}}\left(1+\hat{\chi}_{c}^{*}\right) \dot{\hat{E}}=\frac{\omega^{2} \hat{E}^{*}}{c_{0}^{2}} \hat{\chi}_{c} \text { in } W_{c} \times \mathcal{B} \\
\dot{\Pi}_{a}(t, x)=\frac{\epsilon_{0} \omega_{c}}{\pi^{2}}\left(\frac{1}{2} \Im\left(\hat{\chi}_{c}(x)\right)\left|\int_{W_{c}} \hat{E}^{*}(\omega, x) e^{-i \omega t} d \omega\right|^{2}+\right. \\
\Im\left(\hat{\chi}_{c}^{*}(x)\right) \Re\left[\int_{W_{c}} \dot{\hat{E}}(\omega, x) e^{-i \omega t} d \omega \int_{W_{c}} \frac{\left.\left.\hat{E}^{*}(\omega, x) e^{-i \omega t} d \omega\right]\right) \text { in }(0, T) \times \mathcal{B} .}{} .\right.
\end{array}\right.
$$

Equivalently, with the notation (3.13),

$$
\dot{\Pi}_{a}(t, x)=\frac{\epsilon_{0} \omega_{c}}{2 \pi^{2}} \Im\left(\hat{\chi}_{c}(x)\right)\left|\mathcal{E}^{*}(t, x)\right|^{2}+\frac{\epsilon_{0} \omega_{c}}{\pi^{2}} \Im\left(\hat{\chi}_{c}^{*}(x)\right) \Re\left[\dot{\mathcal{E}}(t, x) \overline{\mathcal{E}^{*}}(t, x)\right] \quad \text { in }(0, T) \times \mathcal{B} .
$$

So

$$
\begin{aligned}
\int_{(0, T) \times \mathcal{B}}\left(\Gamma q^{*}+\zeta^{*}\right) \dot{\Pi}_{a}(t, x) d t d x & =\frac{\epsilon_{0} \omega_{c}}{2 \pi^{2}} \int_{(0, T) \times \mathcal{B}}\left(\Gamma q^{*}+\zeta^{*}\right) \Im\left(\hat{\chi}_{c}(x)\right)\left|\mathcal{E}^{*}(t, x)\right|^{2} d t d x \\
& +\frac{\epsilon_{0} \omega_{c}}{\pi^{2}} \int_{(0, T) \times \mathcal{B}}\left(\Gamma q^{*}+\zeta^{*}\right) \Im\left(\hat{\chi}_{c}^{*}(x)\right) \Re(\dot{\mathcal{E}}(t, x) \overline{\mathcal{E}} *(t, x)) d t d x
\end{aligned}
$$

As the $\operatorname{curl}_{x} \operatorname{curl}_{x}$ operator is self adjoint, performing integration by parts gives

$$
\begin{aligned}
& \int_{(0, T) \times \mathcal{B}}\left(\Gamma q^{*}+\zeta^{*}\right)(t, x) \Im\left(\hat{\chi}_{c}^{*}(x)\right) \dot{\mathcal{E}}(t, x) \overline{\mathcal{E}^{*}}(t, x) d x d t \\
& =\int_{(0, T) \times \mathcal{B}}\left(\Gamma q^{*}+\zeta^{*}\right)(t, x) \Im\left(\hat{\chi}_{c}^{*}(x)\right)\left(\int_{W_{c}} \dot{\hat{E}}(\omega, x) e^{-i \omega t} d \omega\right) \overline{\mathcal{E}^{*}}(t, x) d x d t \\
& =\int_{W_{c} \times \mathcal{B}} \Im\left(\hat{\chi}_{c}^{*}(x)\right)\left(\int_{0}^{T}\left(\Gamma q^{*}+\zeta^{*}\right)(t, x) \overline{\mathcal{E}^{*}}(t, x) e^{-i \omega t} d t\right) \dot{\hat{E}}(\omega, x) d \omega d x \\
& =\int_{W_{c} \times \mathcal{B}}\left(\operatorname{curl}_{x} \operatorname{curl}_{x} \Phi^{*}-\frac{\omega^{2}}{c_{0}^{2}}\left(1+\hat{\chi}_{c}^{*}\right) \Phi^{*}\right)(\omega, x) \dot{\hat{E}}(\omega, x) d \omega d x \\
& =\int_{W_{c} \times \mathcal{B}}\left(\operatorname{curl}_{x} \operatorname{curl}_{x} \dot{\hat{E}}-\frac{\omega^{2}}{c_{0}^{2}}\left(1+\hat{\chi}_{c}^{*}\right) \dot{\hat{E}}\right)(\omega, x) \Phi^{*}(\omega, x) d \omega d x
\end{aligned}
$$




$$
\begin{aligned}
& =\int_{W_{c} \times \mathcal{B}} \frac{\omega^{2} \hat{E}^{*} \Phi^{*}}{c_{0}^{2}}(\omega, x) \hat{\chi}_{c}(x) d \omega d x \\
& =\int_{\mathcal{B}}\left(\int_{W_{c}} \frac{\omega^{2}}{c_{0}^{2}} \hat{E}^{*}(\omega, x) \Phi^{*}(\omega, x) d \omega\right) \hat{\chi}_{c}(x) d x .
\end{aligned}
$$

This gives the result since

$$
\begin{aligned}
& \int_{(0, T) \times \mathcal{B}}\left(\Gamma q^{*}+\zeta^{*}\right)(t, x) \Im\left(\hat{\chi}_{c}^{*}(x)\right) \Re\left(\dot{\mathcal{E}}(t, x) \overline{\mathcal{E}^{*}}(t, x)\right) d t d x \\
& =\Re\left(\int_{(0, T) \times \mathcal{B}}\left(\Gamma q^{*}+\zeta^{*}\right)(t, x) \Im\left(\hat{\chi}_{c}^{*}(x)\right) \dot{\mathcal{E}}(t, x) \overline{\mathcal{E}^{*}}(t, x) d t d x\right) \\
& =\Re\left(\int_{\mathcal{B}}\left(\int_{W_{c}} \frac{\omega^{2}}{c_{0}^{2}} \hat{E}^{*}(\omega, x) \Phi^{*}(\omega, x) d \omega\right) \hat{\chi}_{c}(x) d x\right) \\
& =\int_{\mathcal{B}} \Re\left(\int_{W_{c}} \frac{\omega^{2}}{c_{0}^{2}} \hat{E}^{*}(\omega, x) \Phi^{*}(\omega, x) d \omega\right) \Re\left(\hat{\chi}_{c}(x)\right) d x \\
& -\int_{\mathcal{B}} \Im\left(\int_{W_{c}} \frac{\omega^{2}}{c_{0}^{2}} \hat{E}^{*}(\omega, x) \Phi^{*}(\omega, x) d \omega\right) \Im\left(\hat{\chi}_{c}(x)\right) d x
\end{aligned}
$$

\subsection{The optimality system}

For the regularization term $f$, we find that

$$
\begin{aligned}
\nabla f\left(\hat{\chi}_{c}^{*}\right) \cdot \hat{\chi}_{c} & =\alpha \Re\left(\int_{\mathcal{B}} \overline{\left(\hat{\chi}_{c}^{*}-\hat{\chi}_{c}^{e x}\right)}(x) \hat{\chi}_{c}(x) d x\right) \\
& =\alpha \int_{\mathcal{B}}\left(\Re\left(\hat{\chi}_{c}^{*}-\hat{\chi}_{c}^{e x}\right) \Re\left(\hat{\chi}_{c}\right)+\Im\left(\hat{\chi}_{c}^{*}-\hat{\chi}_{c}^{e x}\right) \Im\left(\hat{\chi}_{c}\right)\right)(x) d x .
\end{aligned}
$$

Thus, putting together the expressions (3.15) and (3.17), we have for every $\hat{\chi}_{c} \in \mathcal{U}_{a d}$

$$
\begin{aligned}
\nabla \mathcal{J}\left(\hat{\chi}_{c}^{*}\right) \cdot\left(\hat{\chi}_{c}-\hat{\chi}_{c}^{*}\right)= & \frac{\epsilon_{0} \omega_{c}}{2 \pi^{2}} \int_{\mathcal{B}}\left\{\left(\int_{0}^{T}\left(\Gamma q^{*}+\zeta^{*}\right)(t, x)\left|\mathcal{E}^{*}(t, x)\right|^{2} d t\right) \Im\left(\hat{\chi}_{c}-\hat{\chi}_{c}^{*}\right)(x)\right. \\
& \left.+2\left(\Re \int_{W_{c}} \frac{\omega^{2}}{c_{0}^{2}} \hat{E}^{*}(\omega, x) \Phi^{*}(\omega, x) d \omega\right) \Re\left(\hat{\chi}_{c}-\hat{\chi}_{c}^{*}\right)(x)\right) \\
& \left.\left.-2\left(\Im \int_{W_{c}} \frac{\omega^{2}}{c_{0}^{2}} \hat{E}^{*}(\omega, x) \Phi^{*}(\omega, x) d \omega\right) \Im\left(\hat{\chi}_{c}-\hat{\chi}_{c}^{*}\right)(x)\right)\right\} d x \\
& +\alpha \int_{\mathcal{B}}\left[\Re\left(\hat{\chi}_{c}^{*}-\hat{\chi}_{c}^{e x}\right) \Re\left(\hat{\chi}_{c}-\hat{\chi}_{c}^{*}\right)+\Im\left(\hat{\chi}_{c}^{*}-\hat{\chi}_{c}^{e x}\right) \Im\left(\hat{\chi}_{c}-\hat{\chi}_{c}^{*}\right)\right](x) d x \geq 0 .
\end{aligned}
$$

Decoupling with respect to real and imaginary parts, we get for any $\left(v_{r}, v_{i}\right)$ real valued functions 
such that $v_{r}+i \Im\left(\hat{\chi}_{c}^{*}\right)=\hat{\chi}_{c} \in \mathcal{U}_{a d}$ and $\Re\left(\hat{\chi}_{c}^{*}\right)+i v_{i}=\hat{\chi}_{c} \in \mathcal{U}_{a d}$

$$
\left\{\begin{array}{l}
\int_{\mathcal{B}} \Re\left(\frac{\epsilon_{0} \omega_{c}}{c_{0}^{2} \pi^{2}} \int_{W_{c}} \omega^{2} \hat{E}^{*}(\omega, x) \Phi^{*}(\omega, x) d \omega+\alpha\left(\hat{\chi}_{c}^{*}-\hat{\chi}_{c}^{e x}\right)(x)\right)\left(v_{r}-\Re\left(\hat{\chi}_{c}^{*}\right)\right)(x) d x \geq 0 \\
\int_{\mathcal{B}}\left\{\frac{\epsilon_{0} \omega_{c}}{2 \pi^{2}}\left(\int_{0}^{T}\left(\Gamma q^{*}+\zeta^{*}\right)(t, x)\left|\mathcal{E}^{*}(t, x)\right|^{2} d t\right)\right. \\
\left.-\Im\left(\frac{\epsilon_{0} \omega_{c}}{c_{0}^{2} \pi^{2}} \int_{W_{c}} \omega^{2} \hat{E}^{*}(\omega, x) \Phi^{*}(\omega, x) d \omega-\alpha\left(\hat{\chi}_{c}^{*}-\hat{\chi}_{c}^{e x}\right)(x)\right)\right\}\left(v_{i}-\Im\left(\hat{\chi}_{c}^{*}\right)\right)(x) d x \geq 0 .
\end{array}\right.
$$

We may now give first order necessary conditions to get a solution of the discretized problem (3.5).

Theorem 3.2. Let $\chi_{c}^{*}$ be an optimal solution of the minimization problem (3.5). Then, the following optimality system is satisfied

- State equations: (3.7) with the corresponding initial and boundary conditions.

- Adjoint equations: (3.11) - (3.12) for pressure and temperature, and (3.14) for electrical field.

- Projection equations: (3.18) (or equivalently (3.19)).

\section{Conclusion}

We have presented what we consider as a complete modelling of the thermoacoustic process with microwave sources. Since the complete model is quite complex, we focused on the very case where the source signal presents a slowly time-varying envelope which is practically relevant. We have presented the inverse and the method to solve it in a formal way. Next steps are the complete theoretical mathematical analysis in an infinite dimensional framework and the numerical experimentation (solving the optimality system). This is challenging since there are many open problems, in particular in terms of the overall numerical burden that this coupling formalism will generate. However the electromagnetic system (in the Fourier space) and the pressure-temperature are slightly coupled. Next issue will be the mathematical study of the pressure-temperature system together with (3.11) - (3.12) and the identification of $\Pi_{a}$ in a first step.

\section{References}

[1] H. Ammari, E. Bossy, V. Jugnon, and H. Kang. Reconstruction of the optical absorption coefficient of a small absorber from the absorbed energy density. SIAM Journal on Applied Mathematics, 71(3):676-693, 2011.

[2] H. Ammari, J. Garnier, W. Jing, and L. H. Nguyen. Quantitative thermo-acoustic imaging: An exact reconstruction formula. J. Differential Equations, 254:1375-1395, 2013.

[3] G. Bal, A. Jollivet, and V. Jugnon. Inverse transport theory of photoacoustics. Inverse Problems, 26(2):025011, 2010. 
[4] G. Bal and K. Ren. Multi-source quantitative photoacoustic tomography in a diffusive regime. Inverse Problems, 27:20p, 2011.

[5] G. Bal, K. Ren, G. Uhlmann, , and T. Zhou. Quantitative thermo-acoustics and related problems. Inverse Problems, 27(5):055007, 2011.

[6] G. Bal and T. Zhou. Hybrid inverse problems for a system of maxwell's equations. Inverse Problems, 30(5):17p, 2014.

[7] M. Bergounioux, X. Bonnefond, T. Haberkorn, and Y. Privat. An optimal control problem in photoacoustic tomography. Mathematical Models and Methods in Applied Sciences, 24(14):2943-48, 2014.

[8] M. J. Burfeindt, T. J. Colgan, R. O. Mays, J. D. Shea, N. Behdad, B. D. Van Veen, and S. C. Hagness. Mri-derived 3d-printed breast phantom for microwave breast imaging validation. IEEE Antennas and Wireless Propagation Letters, 11:1610-1613, 2012.

[9] G. Chen, X. Wang, and Q. Liu. Microwave-induced thermo-acoustic tomography system using TRM-PSTD technique. PIER-B, 48:43-59, 2013.

[10] B. Cox and P.C. Beard. Modeling photoacoustic propagation in tissue using k-space techniques. In L.V. Wang, editor, Photoacoustic Imaging and Spectroscopy, chapter 3, pages 25-34. CRC Press, 2009.

[11] F.A. Duck. Physical Properties of Tissue: A Comprehensive Reference Book. Institution of Physics \& Engineering in Medicine \& Biology, 2012.

[12] P. Elbau, L. Mindrinos, and O. Scherzer. Inverse problems of combined photoacoustic and optical coherence tomography. Math. Methods Appl. Sci., (early view), 2016.

[13] D. Fallon, L. Yan, G. W. Hanson, and S. K. Patch. RF testbed for thermoacoustic tomography. Review of Scientific Instrument, 80:064301, 2009.

[14] S. Gabriel, R.W. Lau, and C. Gabriel. The dielectric properties of biological tissues. II. Measurements in the frequency range $10 \mathrm{~Hz}$ to $20 \mathrm{GHz}$. Physics in Medecine and Biology, 41(11):2251-2269, 1996.

[15] H. Gao, H. Zhao, and S. Osher. Bregman methods in quantitative photoacoustic tomography. Technical Report 10-42, Univ. Calif. Los Angeles UCLA, 2010.

[16] M. Haltmeier, L. Neumann, and S. Rabanser. Single-stage reconstruction algorithm for quantitative photoacoustic tomography. Inverse Problems, 31(6):065005, 2015.

[17] L. Huang, L. Yao, L. Liu, J. Rong, and H. Jiang. Quantitative thermoacoustic tomography: Recovery of conductivity maps of heterogeneous media. Applied Physics Letters, 101(24), 2012 .

[18] J.D. Jackson. Classical Electrodynamics. Wiley, 1998.

[19] R. A. Kruger, W. L. Kiser, D. R. Reinecke, G. A. Kruger, and R. L. Eisenhart. Thermoacoustic computed tomography of the breast at $434 \mathrm{MHz}$. IEEE MTT-S International Microwave Symposium Digest, 2:591-595, 1999. 
[20] R.A. Kruger, W.L. Kiser, K.D. Miller, and H.E. Reynolds. Thermoacoustic CT: imaging principles. Proc. SPIE, 3916:150, 2000.

[21] P. Kuchment and L. Kunyansky. Mathematics of thermoacoustic tomography. European J. Appl. Math., 19:191-224, 2008.

[22] P. Kuchment and L. Kunyansky. Mathematics of photoacoustic and thermoacoustic tomography. In Otmar Scherzer, editor, Handbook of Mathematical Methods in Imaging, pages 1117-1167. Springer New York, New York, NY, 2015.

[23] M. Lazebnik, L. McCartney, D. Popovic, C.B. Watkins, M.J. Lindstrom, J. Harter, S. Sewall, A. Magliocco, J.H. Booske, M. Okoniewski, and S.C. Hagness. A large-scale study of the ultrawideband microwave dielectric properties of normal breast tissue obtained from reduction surgeries. Physics in Medecine and Biology, 52:2637-2656, 2007.

[24] C. Li, M. Pramanik, G. Ku, and L.V. Wang. Image distortion in thermoacoustic tomography caused by microwave diffraction. Physical Review E, 77:31923, 2008.

[25] P. Monk. Finite Element Methods for Maxwell's Equations. Oxford University Press, 2003.

[26] W. Naetar and O. Scherzer. Quantitative photoacoustic tomography with piecewise constant material parameters. SIAM Journal on Imaging Sciences, 7(3):1755-1774, 2014.

[27] L. Nie, D. Xing, D. Yang, L. Zeng, and Q. Zhou. Detection of foreign body using fast thermoacoustic tomography with a multielement linear transducer array. Applied Physics Letters, 90:174109, 2007.

[28] S. K Patch and O.Scherzer. Photo- and thermo-acoustic imaging introduction. Inverse Problems, 6(1-10), 2007.

[29] M. Pramanik, G. Ku, C. Li, and L. V. Wang. Design and evaluation of a novel breast cancer detection system combining both thermoacoustic (ta) and photoacoustic (pa) tomography. Med Phys, 35(6):2218-2223, 2008.

[30] T. Saratoon, T. Tarvainen, B. Cox, and S. Arridge. A gradient-based method for quantitative photoacoustic tomography using the radiative transfer equation. Inverse Problems, $29,2013$.

[31] P. Shao, T. Harrison, and R. J. Zemp. Iterative algorithm for multiple illumination photoacoustic tomography (mipat) using ultrasound channel data. Biomed. Opt. Express., $3: 3240-49,2012$.

[32] N. Song, Deumié C., and A. Da Silva. Considering sources and detectors distributions for quantitative photoacoustic tomography (qpat). Biomed. Opt. Express., 5:3960-74, 2014.

[33] K. Wang and M. A. Anastasio. Photoacoustic and thermoacoustic tomography: Image formation principles. In Otmar Scherzer, editor, Handbook of Mathematical Methods in Imaging, pages 1081-1116. Springer New York, New York, NY, 2015.

[34] L.V. Wang, editor. Photoacoustic Imaging and Spectroscopy, volume 144 of Optical Science and Engineering. CRC Press, 2009. 
[35] L.V. Wang, X. Zhao, H. Sun, and G. Ku. Microwave-induced acoustic imaging of biological tissues. Review of Scientific Instrument, 70(9):3744, 1999.

[36] X. Wang, D. R. Bauer, R. Witte, and H. Xin. Microwave-induced thermoacoustic imaging model for potential breast cancer detection. IEEE Transactions on Biomedical Engineering, 59(10):2782-2791, 2012.

[37] Z. Yuan and H. Jiang. Quantitative photoacoustic tomography. Philos. Trans. R. Soc. -Math. Phys. Eng. Sci., 367:3043-54, 2009.

[38] R. J. Zemp. Quantitative photoacoustic tomography with multiple optical sources. Appl. Opt., 49(18):3566-3572, Jun 2010. 